\title{
Real-Time Respiratory Motion Correction for Cardiac Electrophysiology Procedures Using Image-Based Coronary Sinus Catheter Tracking
}

\author{
YingLiang Ma ${ }^{1}$, Andy P. King ${ }^{1}$, Nicolas Gogin ${ }^{2}$, C. Aldo Rinaldi ${ }^{3}$, \\ Jaswinder Gill ${ }^{3}$, Reza Razavi ${ }^{1}$, and Kawal S. Rhode ${ }^{1}$ \\ ${ }^{1}$ Division of Imaging Sciences, King's College London, SE1 7EH, UK \\ ${ }^{2}$ Medisys Research Laboratory, Philips Healthcare, Paris, France \\ ${ }^{3}$ Department of Cardiology, Guy's \& St. Thomas' Hospitals NHS Foundation Trust, London, \\ SE1 7EH, UK \\ $\mathrm{y} \cdot \mathrm{ma} \mathrm{kcl} \cdot \mathrm{ac} \cdot \mathrm{uk}$
}

\begin{abstract}
X-ray fluoroscopically guided cardiac electrophysiological procedures are routinely carried out for diagnosis and treatment of cardiac arrhythmias. X-ray images have poor soft tissue contrast and, for this reason, overlay of static 3D roadmaps derived from pre-procedural volumetric data can be used to add anatomical information. However, the registration between the 3D roadmap and the 2D X-ray data can be compromised by patient respiratory motion. We propose a novel method to correct for respiratory motion using real-time image-based coronary sinus (CS) catheter tracking. The first step of the proposed technique is to use a blob detection method to detect all possible catheter electrodes in the Xray data. We then compute a cost function to select one CS catheter from all catheter-like objects. For correcting respiratory motion, we apply a low pass filter to the $2 \mathrm{D}$ motion of the CS catheter and update the 3D roadmap using this filtered motion. We tested our CS catheter tracking method on 1048 fluoroscopy frames from 15 patients and achieved a success rate of $99.3 \%$ and an average 2D tracking error of $0.4 \mathrm{~mm} \pm 0.2 \mathrm{~mm}$. We also validated our respiratory motion correction strategy by computing the $2 \mathrm{D}$ target registration error (TRE) at the pulmonary veins and achieved a TRE of $1.6 \mathrm{~mm} \pm 0.9 \mathrm{~mm}$.
\end{abstract}

\section{Introduction}

Cardiac electrophysiological (EP) procedures are traditionally carried out under X-ray fluoroscopic guidance to diagnose and treat cardiac arrhythmias. However, X-ray images have poor soft tissue contrast and it is difficult to interpret the anatomical context directly from these images. To overcome the lack of soft tissue contrast, a three-dimensional (3D) roadmap can be generated from 3D high-resolution computed tomography $(\mathrm{CT}) /$ magnetic resonance images (MRI), registered and overlaid in realtime with X-ray fluoroscopy images [1]. Currently, the 3D roadmap remains static and does not move with the patient's respiratory motion. In some cases, respiratory motion can cause a two-dimensional (2D) registration error of over $14 \mathrm{~mm} \mathrm{[2],} \mathrm{which}$ 
is a significant compromise in the accuracy of guidance. A number of groups have previously addressed the issue of respiratory motion correction for cardiac interventions. Motion-compensated navigation for coronary interventions based on magnetic tracking was suggested in [3], but it required additional special hardware. Several image-based approaches have been developed that use only information from the $\mathrm{X}$ ray fluoroscopic images themselves. Shechter et al. [4] constructed a model of cardiac and respiratory motion of the coronary arteries from biplane contrast-enhanced X-ray image sequences. The model was applied by tracking the motion of the diaphragm in subsequent (non-enhanced) X-ray images. However, forming the model from X-ray images under contrast injection means that it will be constructed from a limited amount of data. Furthermore, the diaphragm is not always in the X-ray field of view, particularly for obese patients. Brost et al. [5] developed an image-based respiratory motion correction method for EP procedures by tracking the $3 \mathrm{D}$ position of a lasso catheter from biplane X-ray images. Unlike tracking the diaphragm, this method directly tracks an instrument very close to the target region of the EP procedure. However, it also has some limitations. Firstly, the lasso catheter is particular for only a subset of EP procedures and it does not always remain stationary inside the heart. Secondly, the majority of X-ray systems are monoplane systems. Finally, the maximum frame rate of the lasso catheter tracking was only 3 frames per second and the tracking method required manual initialization.

In this paper, we present a novel method to track the coronary sinus (CS) catheter from X-ray images in real-time. The reason we choose the CS catheter is that it is almost ubiquitously present during EP procedures. The CS catheter has several electrodes which are highly visible in normal dose and low dose X-ray images. Furthermore, the CS catheter remains in place throughout the procedure, its position is not routinely altered and it is normally not close to other catheters. Our technique works by first using a fast multi-scale blob detection method to detect all possible electrodelike objects in the X-ray image. The main novelty of our approach is that we then use prior knowledge of the CS catheter geometry to identify a single CS catheter from all of these candidate objects. This allows us to quickly and reliably distinguish the CS catheter from other catheters and instruments in the X-ray image. Our tracking method uses the whole image as a region of interest so that we are able to track the CS catheter even in the presence of large sudden motion. Furthermore, the proposed method does not require any user interaction. We apply the technique to correct respiratory motion during cardiac EP procedures by applying filtered $2 \mathrm{D}$ in-plane translational motion to the $3 \mathrm{D}$ roadmap.

\section{Method}

CS catheters are often used as diagnostic catheters and one of most commonly used CS catheters is the 10-electrode catheter. The arrangement of electrodes has two variations. One is evenly distributed and the other is paired (figure 1a). The presence of 10 electrodes is the unique feature which we use to separate the CS catheter from other catheters, instruments and ECG leads. The first step of our proposed method is to run a blob detection method to detect all possible electrode-like objects. 


\subsection{Blob Detection}

We use a multi-scale blob detector based on the determinant of the Hessian matrix [6]. We consider a continuous function of two variables such that the value of the function at $(x, y)$ is given by $f(x, y)$. Then the determinant of the Hessian matrix is defined as

$$
\operatorname{det} H(f(x, y))=f_{x x} f_{y y}-f_{x y}^{2}
$$

where $f_{x x}, f_{y y}$ and $f_{x y}$ are the second-order partial derivates of the function $f(x, y)$. Blobs can be defined as local extrema of the determinant of the Hessian matrix. To translate this theory to work with $2 \mathrm{D}$ images, we first replace the function $f(x, y)$ with discrete image pixel intensities $I(x, y)$. Then we calculate the derivatives by convolving the image with second order Gaussian derivative masks (figure 1b).

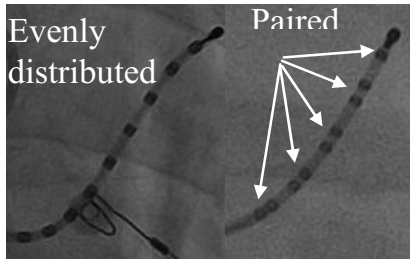

(a)

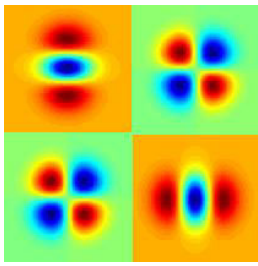

(b)

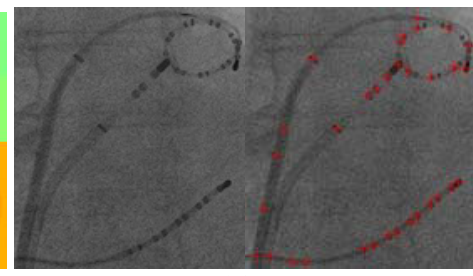

(c)

Fig. 1. a) Two variations of 10 electrode CS catheter. b) Gaussian derivative masks. c) The result from the blob detection method. Red crosses are the positions of electrode-like blobs.

To introduce a scale factor $t$, we first introduce the scale-space representation $L(x, y ; t)$ of the image $I(x, y)$ by $L(x, y ; t)=I(x, y) * g(x, y ; t)$, where * is the convolution operator and the Gaussian filter $g(x, y ; t)$ is defined as $g(x, y ; t)=\frac{1}{2 \pi t} e^{-\left(x^{2}+y^{2}\right) / 2 t}$. Here scale factor $t=\sigma^{2}$ where $\sigma$ is the standard deviation of the Gaussian function. Now we can derive the scale-normalized determinant of the Hessian from eq. (1) and we get

$$
\operatorname{det} H(L(x, y ; t))=t^{2}\left(L_{x x} L_{y y}-L_{x y}^{2}\right)
$$

Here $L_{x x}=I(x, y)^{*} g_{x x}$ where

$$
g_{x x}(x, y ; t)=-\frac{1}{2 \pi t^{2}}\left(1-\frac{x^{2}}{t}\right) e^{-\left(x^{2}+y^{2}\right) / 2 t}
$$

Similar definitions apply for $L_{y y}$ and $L_{x y}$. These Gaussian derivatives ( $g_{x x}$, $g_{y y}$ and $g_{x y}$ ) are often known as Laplacian of Gaussians (LoG). In practice, we just pre-compute the masks of these Gaussian derivatives (figure 1b), convolve with the input image and calculate the determinant of the Hessian matrix using eq. (2). Blobs 
are detected as regional maxima of the determinant of the Hessian matrix and we also define the strength of the blob as the normalized value of the determinant of the Hessian matrix.

Finally, we need to decide the scale factors for detecting all electrodes on the CS catheter. As shown in figure 1a, there are only two sizes of electrodes. One is a big catheter tip electrode and the others are small electrodes. Therefore, two fixed scale factors are sufficient which could lead to a more efficient and robust implementation than other automatic scale selection methods. To calculate the scale factor $t$, we use $t=((s-1) / 3)^{2}$ where $s$ is the size of blob. This equation is motivated by the " $3 \sigma$ " ( $t=\sigma^{2}$ ) rule that $99 \%$ of energy of the Gaussian is within three standard deviations and was empirically tested for our application. Figure 1c demonstrates the result of blob detection in a low-dose X-ray image. The strength of the blob is dependent on the blob size. The value of the blob size, $s$, was set to 6 and 3 pixels for the catheter tip electrode and the smaller electrodes, respectively.

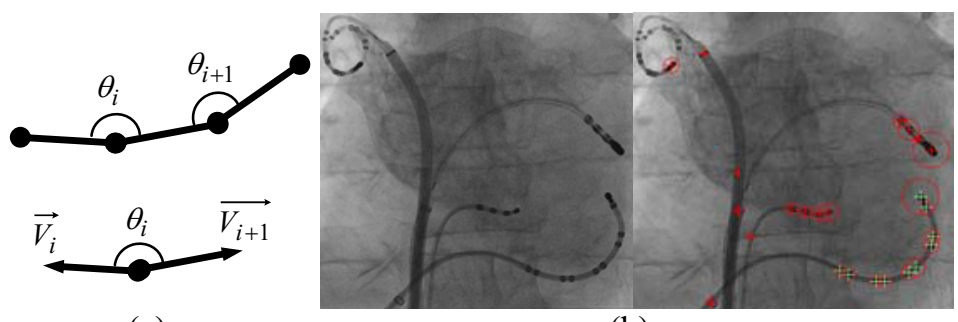

(a)

(b)

Fig. 2. a) Definition and calculation of the deviation angle. b) The result from the CS catheter detection method. Green crosses are the positions of CS catheter electrodes. Red crosses are the positions of other catheter electrodes. The size of the red circles represents the strength of the blobs.

\subsection{Catheter Detection}

The next step involves deciding which combination of blobs represents the CS catheter. We select the 50 highest strength blobs from the blob detection method. Each blob is connected to its nearest neighbour. Based on the shape model of the 10electrode CS catheter we design a cost function to estimate the likelihood of candidate catheter-like objects. Catheter-like objects should have a large blob as the catheter tip and several smaller blobs after it. As we know there are two variations of the arrangement of electrodes (figure 1a). We designed a unified cost function. The cost function consists of two parts. One is the smooth variation of deviation angle between two consecutive edges (figure 2a). The other is the smooth variation of the strength difference between two consecutive blobs except the catheter tip electrode. We exclude the catheter tip electrode from the calculation of the cost function because the strength of the response of the blob detector is dependent on the blob size. Therefore, the catheter tip electrode would have a higher weight in the cost function. The cost function is defined as follows 


$$
\text { CSCathCost }=\frac{\sum_{i=1}^{N-2}\left|\cos \theta_{i}-\overline{\cos \theta}\right|}{N-2}+\frac{\sum_{i=2}^{N}\left|B_{l o b_{i}}-\overline{\text { Blob }}\right|}{N-1}
$$

where $\overline{\cos \theta}$ is the mean of cosines of all deviation angles and $\overline{B l o b}$ is the mean of blob strengths of the 9 electrodes (excluding the catheter tip electrode). Blob $i$ is normalized to a range of zero to one which is the same value range as $\cos \theta_{i} . N$ is the total number of electrodes ( $N=10$ in our case). $\cos \theta_{i}$ can be computed efficiently using $\cos \theta_{i}=\vec{V}_{i} \bullet \overrightarrow{V_{i+1}} /\left(\left\|\vec{V}_{i}\right\| \cdot\left\|\overrightarrow{V_{i+1}}\right\|\right)$, where $\bullet$ is the dot product and \|\| is the vector length. $\vec{V}_{i}$ and $\overrightarrow{V_{i+1}}$ are defined in figure $2 \mathrm{a}$.

To reduce the number of catheter candidates, we introduce two constraints to quickly remove unwanted candidates. The first constraint is the maximum electrode gap. Currently, we define it as twice the length of the maximum distance Max dist between two neighbouring CS catheter electrodes (we obtained Max dist by physical measurement of a CS catheter). 10-electrode CS catheters have two variations of electrode distributions and we choose whichever maximum distance between two neighbour electrodes is larger. The second constraint is the minimum deviation angle which must be larger than 90 degrees as catheters in human vessels could not have sharp turns. So, this condition can be translated into $\overrightarrow{V_{i}} \bullet \overrightarrow{V_{i+1}}<0$. The overall algorithm for detecting one 10-electrode CS catheter is presented in Algorithm 1. Figure $2 \mathrm{~b}$ gives an example CS catheter detection result in one clinical X-ray image.

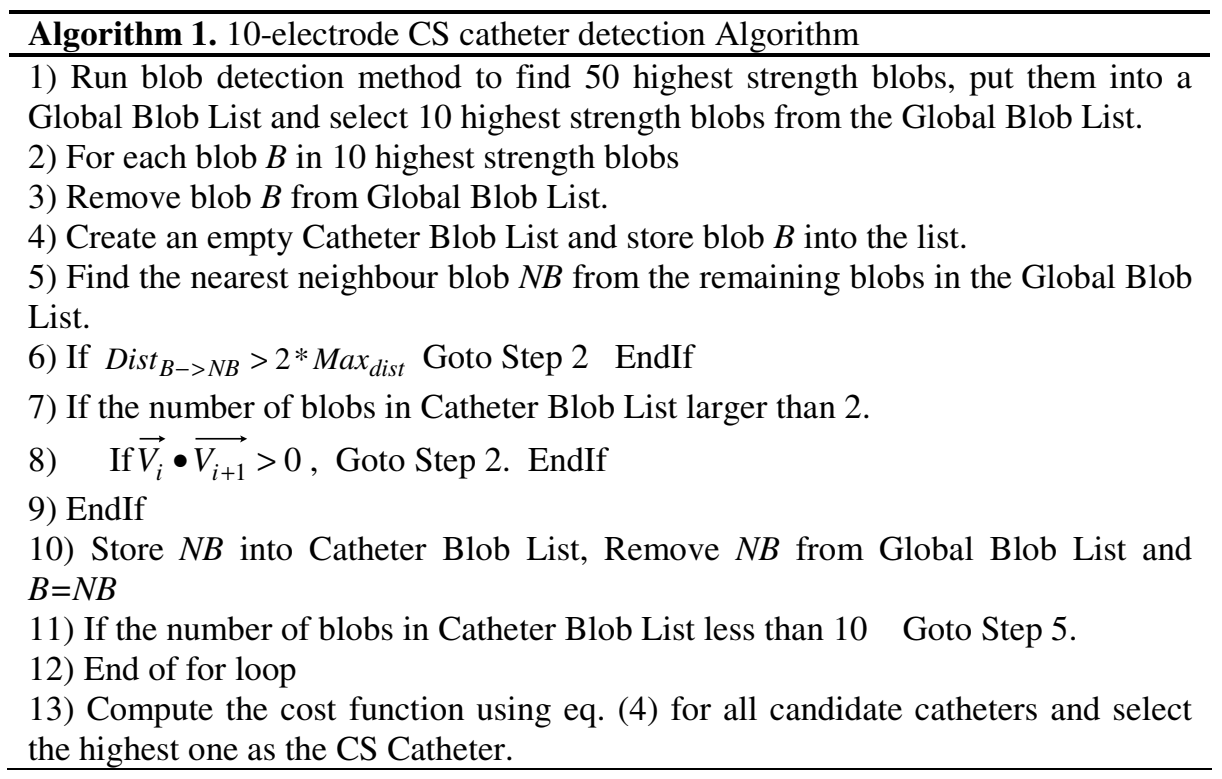




\subsection{Motion Correction}

We apply our CS detection method to correct for respiratory motion in 2D fluoroscopic image sequences during cardiac EP procedures. CS catheters have been previously used in computing the initial registration for image-guided cardiac EP procedures [7] because they are known to be relatively stable in position with respect to the anatomy. Similarly, our approach tracks the position of the proximal electrode of the 10-electrode CS catheter to use for respiratory motion correction. The reason for choosing this electrode (the distal electrode is the catheter tip) is that the proximal electrode has less cardiac cycle motion and has lower tracking errors than the distal electrode (see section 3.1). For a monoplane X-ray system, we first apply a low pass filter to the $2 \mathrm{D}$ translational motion of the proximal electrode to reduce cardiac motion further and then apply the filtered 2D translational motion directly to the $3 \mathrm{D}$ roadmap.

\section{Results}

We tested the proposed CS catheter tracking method on 1048 clinical X-ray images. There were a total of 18 different clinical fluoroscopy sequences which came from 15 clinical cardiac catheterization cases. $28 \%$ of the clinical X-ray images that we tested were low dose and contained high frequency noise. 2 clinical cases (136 images) used evenly distributed 10-electrode CS catheters and the other 13 cases (912 images) used paired 10-electrode CS catheters. Typically, the clinical X-ray images contained one CS catheter, one ablation catheter and sometimes one lasso catheter. Some X-ray fluoroscopy images, which were acquired during pace-maker implant cases, contained ECG electrodes and wires, and also sternal wire loops.

\subsection{Accuracy, Robustness and Efficiency Test}

Table 1. 2D electrodes detection errors in $\mathrm{mm}$ (excluding partial failure images and electrode 1 is the distal electrode)

\begin{tabular}{|l|c|c|c|c|c|c|c|c|c|c|}
\hline Electrode & 1 & 2 & 3 & 4 & 5 & 6 & 7 & 8 & 9 & 10 \\
\hline Low dose images & 0.4 & 0.6 & 0.3 & 0.3 & 0.3 & 0.3 & 0.3 & 0.3 & 0.3 & 0.3 \\
\hline Normal dose images & 0.4 & 0.8 & 0.3 & 0.4 & 0.3 & 0.3 & 0.4 & 0.4 & 0.3 & 0.3 \\
\hline
\end{tabular}

To test the accuracy of our method, we asked two clinical experts to manually pick the centre of all electrodes of the CS catheter on all 1048 images. Then we calculated the $2 \mathrm{D}$ distance between the manually defined centres and the centres detected by our method for all 10 electrodes. Measurements were made in pixel space and then converted to $\mathrm{mm}$ space using the magnification factor available from the DICOM header of the X-ray data. Overall, we achieved a 2D detection error of $0.39 \mathrm{~mm} \pm 0.22 \mathrm{~mm}$ for all electrodes in all images and we obtained a very similar error of $0.36 \mathrm{~mm} \pm 0.21$ $\mathrm{mm}$ for low dose X-ray images only. The errors of individual electrodes are given in table 1. It is noticeable that electrode No 2 has larger errors than others. This is 
because the second electrode is very close to the catheter tip (the distal electrode) and it could be detected together with the catheter tip as a single large blob.

Our method is robust in low dose images as the errors in low dose images were very similar to the errors in normal dose images. We only observed the partial failure of detection in 8 frames of normal dose images because one of electrodes was occluded by ECG electrodes. We classify a partial failure when the detection method correctly detected the CS catheter but any of electrodes had an error of more than 5 $\mathrm{mm}$. The choice of $5 \mathrm{~mm}$ is motivated by the size of the smallest target structures for EP procedures (the pulmonary veins, approximately $5 \mathrm{~mm}$ in radius). There were no complete failure cases which did not detect the correct CS catheter. The overall success rate of detection was $99.3 \%$.

The major computational load of our method is the blob detection algorithm. We implemented it on the Intel Integrated Performance Primitives Library for fast image convolution calculation. Our method currently achieves a frame rate of 21 frames-persecond using a single-threaded CPU implementation. The performance was evaluated on an Intel Core 2 Duo 2.0GHz laptop with an nVidia Quadro FX 350M graphics card.

\subsection{Motion Correction Results}

The intended application of our CS catheter tracking technique is to update the position of a 3D roadmap. Therefore, as further validation we computed the target registration error (TRE) at the pulmonary veins (PVs), which are the main structures of interest in many EP procedures. For the cases in which the lasso catheter was used for validation of accuracy, it remained stable in one of the pulmonary veins for all the $\mathrm{X}$ ray frames evaluated. We applied the computed 2D translation determined from the CS catheter tracking to the position of the lasso catheter which acts as a surrogate for the position of the PVs since it is rigidly placed within these structures during the procedure. The TRE was then computed as the distance error between this predicted position and the actual position of the lasso catheter in the X-ray data. We calculated the TRE at the PVs on 565 fluoro images (7 patients) and achieved a 2D accuracy of $1.6 \mathrm{~mm} \pm 0.9 \mathrm{~mm}$. The TRE before motion correction was $6.1 \pm 1.8 \mathrm{~mm}$. Figure $3 \mathrm{~b}$

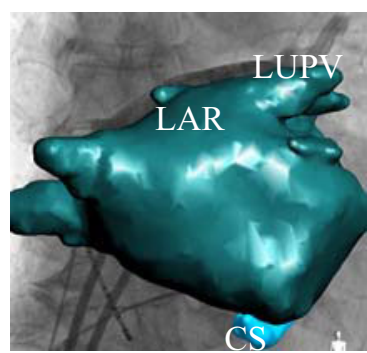

(a)

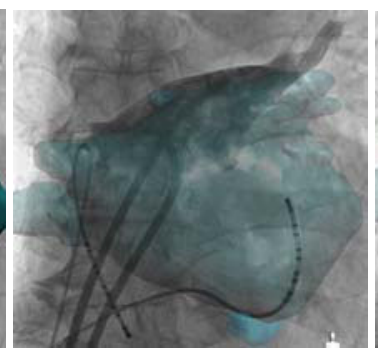

(b)

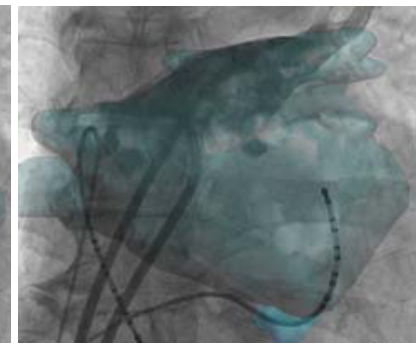

(c)

Fig. 3. Illustrates an MRI overlay on to x-ray fluoroscopy. The initial registration was done using multiple view X-ray acquisitions followed by manual alignment. a) b). Frame of a nonmotion compensated sequence with fluoroscopy overlay. c) Motion compensated fluoroscopy overlay. 
presents an example of a fluoroscopy overlay with respiratory motion correction. In the $3 \mathrm{D}$ roadmap, LAR is the left atrium roof and LUPV is the left upper pulmonary vein. From figure $3 \mathrm{a}$ and $3 \mathrm{~b}$, there is a noticeable mis-alignment between the $3 \mathrm{D}$ roadmap and the X-ray angiography, particularly in the area of LAR and LUPV. The roadmap and X-ray angiography are well matched and the CS is aligned with the CS catheter in figure $3 \mathrm{c}$.

\section{Conclusion and Discussions}

We have developed an accurate and robust method for real-time CS catheter tracking in X-ray fluoroscopy images and applied it to correct respiratory motion in X-ray fluoroscopy image-guided EP procedures. Our tracking method remains robust and accurate even in low dose fluoroscopy images as CS catheter electrodes remain highly visible. Because the majority of normal dose images we tested had fast-changing background contrast (contrast agent injection), our results showed less tracking errors in low dose images than normal dose ones as the accuracy of blob detection is influenced by contrast agent injection. We achieved a sub-millimeter accuracy of our method as the average tracking error for all CS catheter electrodes is $0.4 \mathrm{~mm}$ and maximum error is $0.8 \mathrm{~mm}$. Updating the $3 \mathrm{D}$ roadmap by the filtered $2 \mathrm{D}$ motion of the CS catheter can significantly improve the accuracy of fluoroscopy overlays for cardiac EP procedures. Our CS catheter tracking method has several advantages. First, it is real-time so that as well as being used to detect respiratory motion it could potentially also be applied to the detection of the much faster cardiac cycle motion. Secondly, it does not require any user interaction and can detect the CS catheter position without defining a region of interest in the X-ray image. Finally, it has potential application in more types of cardiac catheterization procedure, rather than only for EP procedures. Other potential applications of our CS catheter detection method that we plan to investigate include gating cardiac and respiratory motion in 3D C-Arm CT sequences to improve the quality of the reconstructed 3D volumes. Also, we plan to investigate its use for 3D real-time registration between the CS catheter and the main branch of the CS geometry in the 3D roadmap. This could lead to a real-time solution to the problem of bulk patient motion. Because our CS catheter tracking method is based on blob detection, it can be extended to simultaneously track a CS catheter and an ablation catheter in real-time. This opens the possibility to design a system to automatically tag ablation positions with motion correction in real-time.

\section{References}

1. Rhode, K.S., Hill, D.L.G., Edwards, P.J., Hipwell, J., Rueckert, D., Sanchez-Ortiz, G., Hegde, S., Rahunathan, V., Razavi, R.: Registration and tracking to integrate X-ray and MR images in an XMR facility. IEEE Transactions on Medical Imaging 24(11), 810-815 (2003)

2. King, A.P., Boubertakh, R., Rhode, K.S., Ma, Y.L., Chinchapatnam, P., Gao, G., Tangcharoen, T., Ginks, M., Cooklin, M., Gill, J.S., Hawkes, D.J., Razavi, R.S., Schaeffter, T.: A subject-specific technique for respiratory motion correction in image-guided cardiac catheterisation procedures. Med. Image Anal. 13(3), 419-431 (2009) 
3. Timinger, H., Krueger, S., Dietmayer, K., Borgert, J.: Motion Compensated Coronary Interventional Navigation by Means of Diaphragm Tracking and Elastic Motion Models. Phys. Med. Biol. 50(3), 491-503 (2005)

4. Shechter, G., Shechter, B., Resar, J.R., Beyar, R.: Prospective motion correction of X-ray images for coronary interventions. IEEE Transactions on Medical Imaging 24(4), 441-450 (2005)

5. Brost, A., Liao, R., Hornegger, J., Strobel, N.: 3-D respiratory motion compensation during EP procedures by image-based 3-D lasso catheter model generation and tracking. In: Yang, G.-Z., Hawkes, D., Rueckert, D., Noble, A., Taylor, C. (eds.) MICCAI 2009. LNCS, vol. 5761, pp. 394-401. Springer, Heidelberg (2009)

6. Tony, L.: Detecting salient blob-like image structures and their scales with a scale-space primal sketch: a Method for focus-of-attention. International Journal of Computer Vision 11(3), 283-318 (1993)

7. Sra, J., Krum, D., Belanger, B., Vaillant, R.: Registration of three dimensional left atrial computed tomographic images with fluoroscopy. Heart Rhythm 2(9), 1020 (2005) 